\title{
Superconductive Magnetic Energy Storage (SMES) External Fields and Safety Considerations
}

\author{
C. Polk \\ Department of Electrical Engineering \\ University of Rhode Island \\ Kingston, Rhode Island - USA
}

\author{
R. W. Boom and Y. M. Eyssa \\ Applied Superconductivity Center \\ University of Wisconsin \\ Madison, Wisconsin - USA
}

\begin{abstract}
This paper addresses preferred SMES configurations and the external magnetic fields which they generate. Possible biological effects of fields are reviewed briefly. It is proposed that SMES units be fenced at the 10 gauss $(1 \mathrm{mT})$ level to keep unrestricted areas safe, even for persons with cardiac pacemakers. For a full size $5000 \mathrm{MWh}\left(1.8 \times 10^{13} \mathrm{~J}\right) \mathrm{SMES}$ the magnetic field decreases to 10 gauss at a radial distance of $2 \mathrm{~km}$ from the center of the coil. Other considerations related to the environmental impact of large SMES magnetic fields are discussed briefly.
\end{abstract}

\section{HISTORY OF SMES}

The technological basis for Superconductive Magnetic Energy Storage (SMES) systems had its beginnings in 1911 when in the course of investigating normal conductors at low temperature Kammerlingh Onnes discovered superconductivity. The ground work for SMES research was laid in the 1960's by several U. S., Japanese, and European groups. ${ }^{1-7}$ Stekly $^{1}$ in 1963 was the first to offer a comprehensive design, which stressed structural problems. In 1971 Peterson and Boom ${ }^{8-9}$ invented the SMES system used today that consists of a superconductive storage coil charged and discharged by an ac/dc Graetz bridge. Starting with the invention in 1971 the Applied Superconductivity Center of the University of Wisconsin has undertaken a broad program in all phases of research, development and design of SMES for electric utility use. Environmental effects of large SMES magnetic fields have been discussed previously by the authors. ${ }^{10}$

\section{CONFIGURATIONS}

Energy storage coil configurations can be single solenoids, groups of two or more solenoids with canceling external fields, or toroids for zero external field. Solenoids are relatively simple and light-weight. Significant design efforts have been devoted by the UW group to utility, accelerator and space applications. These studies show that low aspect ratio solenoids (aspect ratio $\beta$ is solenoid height $\div$ diameter) are superior to other configurations for large SMES applications. ${ }^{11,12}$ Figure 1 shows the proposed low aspect SMES solenoid. The SMES low aspect ratio solenoid designs at UW incorporate a trench in which the solenoidal superconducting magnet is buried to provide cost-effective mechanical support for the coil. A mechanical support frame imbedded in soil or rock transmits magnetic forces from the magnet to the trench wall.

\section{EXTERNAL FIELD OF A $5000 \mathrm{MWh}$ ENERGY STORAGE} SOLENOID

The external field of a SMES solenoid is given by a simple expression in terms of energy stored $E$ and midplane field $B_{M} \cdot{ }^{13}$ At large distances the field components $B_{r}$ and $B_{\theta}$ in spherical coordinates are:

$$
\begin{aligned}
& B_{r}=\frac{\mu_{0}}{\pi} K_{s}(\beta) \frac{E}{B_{M}} \frac{\cos \theta}{r^{3}} \\
& B_{\theta}=\frac{\mu_{0}}{\pi} K_{s}(\beta) \frac{E}{B_{M}} \frac{\sin \theta}{2 r^{3}}
\end{aligned}
$$

where $K_{s}(\beta)$ is a shape factor that approaches unity for $\beta \gg 1$. $K_{s}$ vs. $\beta$ is plotted in Fig. 2. A low $\beta$ solenoid has a larger stray field than a high aspect ratio solenoid. Figure 3 is a plot of the magnetic field contours for a $5000 \mathrm{MWh}\left(1.8 \times 10^{13} \mathrm{~J}\right)$ solenoid. Figure 4 shows the SMES facility layout at the 100 $(10 \mathrm{mT})$ and $10(1 \mathrm{mT})$ gauss lines.

The maximum possible time rate of change of the field at any particular point in space is given by the relation between maximum coil current I, flux density B at the particular point, and the maximum time rate of change of $\mathrm{I}$. Thus if $\mathrm{B}=\mathrm{kI}$, $(d B / d t)=k(d I / d t) . D I / d t$ is about $150 \mathrm{~A} / \mathrm{s}$ during emergency energy dumps and only 3 to $10 \mathrm{~A} / \mathrm{s}$ in normal utility use for I $=50 \times 10^{3} \mathrm{~A}$. The maximum value of $(\mathrm{dB} / \mathrm{dt})$ at points where $B=1 \mathrm{~T}$ is $3 \times 10^{-3} \mathrm{~T} / \mathrm{s}$. At the exclusion fence $(\mathrm{R}=2000 \mathrm{~m})$, where $\mathrm{B}=10 \mathrm{G}=10^{-3} \mathrm{~T}$ we obtain $\mathrm{k}=2 \times 10^{-8}$ and $(\mathrm{dB} / \mathrm{dt})$ 


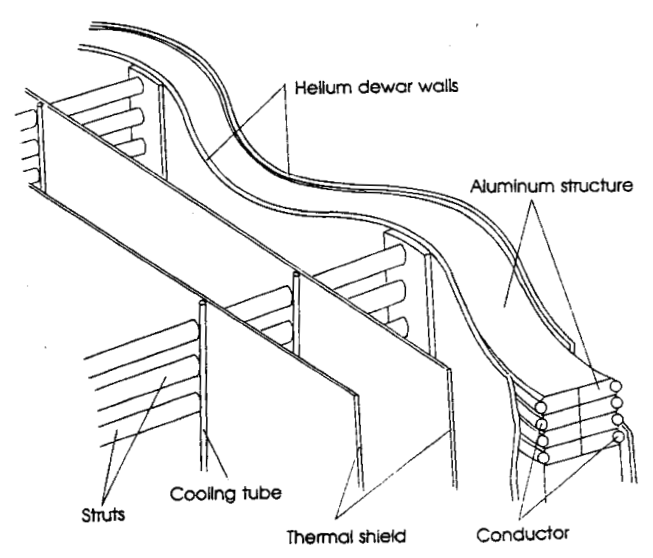

Figure 1 . View of the proposed rippled low aspect ratio solenoid

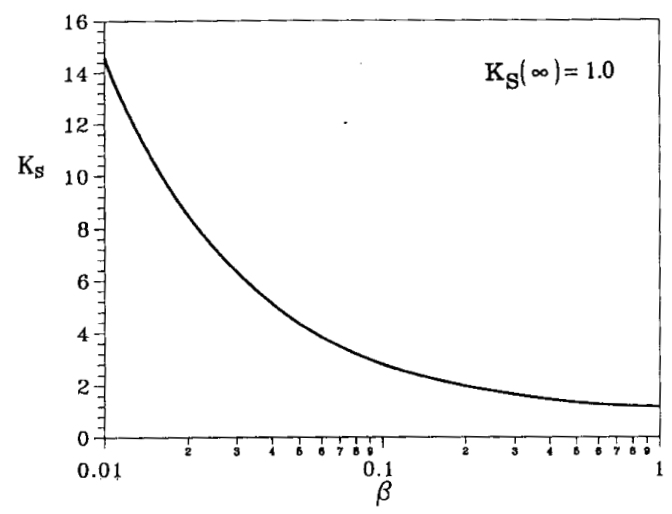

Figure 2. Stray field factor $K_{\mathrm{s}}$ vs. $\beta$.

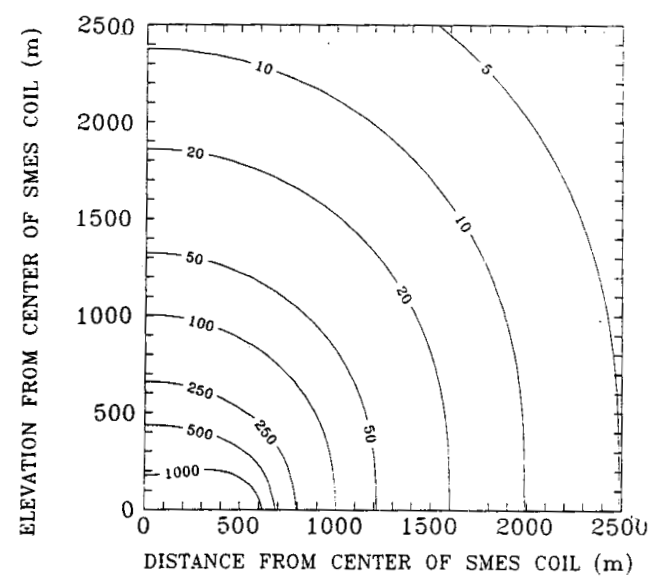

Figure 3. Extemal Fieid of a $5000 \mathrm{MWh}$ SMES (Constant field contours given in Gauss)

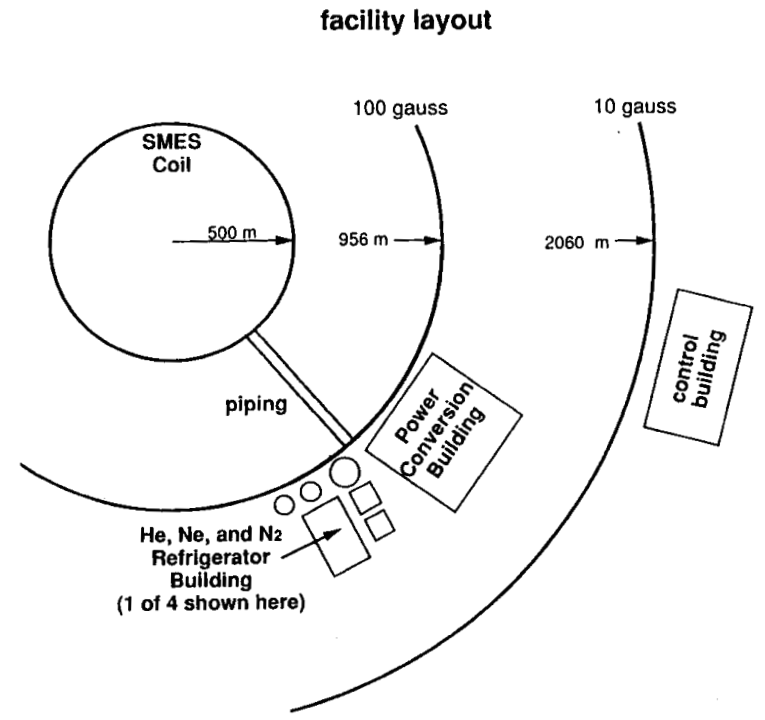

Figure 4. 5000 MWh SMES Facility Layout

$=3.0 \times 10^{-6} \mathrm{~T} / \mathrm{s}$. For comparison we consider $(\mathrm{dB} / \mathrm{dt})$ due to the 1 milligauss $=10^{-7} \mathrm{~T}$ power frequency magnetic field that is present in a typical American home under "low power conditions" (when all major appliances are shut off). Since at $60 \mathrm{~Hz}(\mathrm{~dB} / \mathrm{dT})$ which would be present continuously, or would be exceeded, in this "low power" home, is $3.77\left(10^{-5} \mathrm{~T} / \mathrm{s}\right)$ about ten times higher than that produced by the $5000 \mathrm{MWh}$ at the exclusion fence during the most rapid load change.

\section{SHIELDING}

Three options have been considered for shielding humans and other life forms from the large fringe fields associated with SMES devices. These options, alone and in various combinations, include (1) passive shielding with iron, (2) active shielding with a compensating magnetic coil, and (3) substantial land procurement around an SMES facility. The first two options are generally not economically feasible, whereas the third option is economically attractive in regions where land procurement costs are low. However, in some cases this option could ultimately lead to licensing difficulties because of the intrinsic and/or projected value of land, even in relatively undeveloped areas. Also, the siting of an SMES system within a large exclusion zone addresses only the issue of shielding the radial fringe fields. The vertical fringe fields in the atmosphere and their potential impact on avian species would remain as an environmental issue if land procurement were to be the only shielding option that was implemented. For this reason a combination of the second and third options may need to be 
considered in certain special circumstances. However, the construction of a shield coil would represent a substantial capital investment, and the feasibility of using this option must be weighed carefully against the perceived risk of not shielding the SMES fringe field.

\section{ETM SAFETY PROCEDURES}

The Health and Safety Manual of the University of California's Lawrence Livermore National Laboratory ${ }^{14}$ gives the following "Guidelines" for exposure to DC magnetic fields:

"Maximum Exposure: Workers must never be exposed to fields exceeding 2 Tesla, regardless of the duration of the exposure or the body part exposed. $(1 \mathrm{~T}=10,000 \mathrm{G})$.

Fields Less Than $500 \mathrm{mT}$ : If the peak field to which workers are exposed is less than $500 \mathrm{mT}$, personnel may be exposed to a week-long maximum average field strength of no more than $60 \mathrm{mT}$ (measured at the torso) or $600 \mathrm{mT}$ (measured at the extremities).

Fields Greater Than $500 \mathrm{mT}$ : If the peak exposure is greater than $500 \mathrm{mT}$, workers should be exposed to a daily maximum average field strength of no more than $60 \mathrm{mT}$ (measured at the torso) or $600 \mathrm{mT}$ measured at the extremities."

In the introduction to the guidelines, the Manual ${ }^{14}$ states that "Recent research suggests that some health and genetic problems may be linked to prolonged exposure to strong time-varying magnetic fields. However, such evidence does not exist for steady-state (non-varying) magnetic fields... As a precautionary measure, we list the following standards... for exposure to steady-state magnetic fields...."

Additionally the following more detailed rationale for the DC standards is given:

"Mice have been exposed to fields as strong as $9 \mathrm{~T}$ for short periods of time, and as strong as $1 \mathrm{~T}$ for a lifetime (about 2 years), without evidence of ill effects. One observed consequence of exposure to magnetic fields is that the blood pumped through the aorta generates a voltage increase by magnetohydrodynamic (MHD) action. This small voltage increase appears on an electrocardiogram, and disappears as soon as the animal is removed from a magnetic field. The significance of the MHD effect is not known at this time, but the best available medical advice suggests to limit this MHD voltage to one millivolt. Our standards listed above are designed to fit this requirement."

"In order to assure that the weekly average exposure does not exceed $500 \mathrm{mT}$, exposure to strong fields must be controlled on a day-to-day basis, while exposures to weak fields may be considered in weekly intervals. Consequently, two average-exposure guidelines are given above, one for situations with peak fields greater than $500 \mathrm{mT}$ and the other for fields less than $500 \mathrm{mT}$. Because the heart is the principal organ which may be affected by magnetic fields, the limit to torso exposures is about ten times less than the limit for the extremities."

Guidelines for the clinical use of Magnetic Resonance Imaging (MRI) equipment published by the U.S. Federal Food and Drug Administration ${ }^{15}$ are considerably less cautious and less restrictive, presumably to allow maximal use of the medically beneficial aspects of MRI. The following statements are reproduced from the FDA document ${ }^{15}$ omitting standards for RF heating and acoustic noise which are of no concern in SMES:

"If levels cited below are to be exceeded, the manufacturer must provide valid scientific evidence to establish the safety of operating at intended levels.

o - Static Magnetic Field ( $\left.B_{0}\right)$

Static magnetic field strengths not exceeding $2.0 \mathrm{~T}$ are below the level of concern for the static magnetic field.

o - Rate of Magnetic Field Strength Change with time (dB/dt) Three alternate approaches are described below. Option A is the level of concern below which clearly there is no apparent concern for the safety of the subject. This is the level which has been employed by the Agency in previous PMA submissions. Option B permits the sponsor to utilize values of $\mathrm{dB} / \mathrm{dt}$ that may exceed the $6 \mathrm{~T} / \mathrm{s}$ vaiue but evidence of the actual level achieved will undergo more stringent review. Option $\mathrm{C}$ permits the sponsor to use peripheral nerve stimulation as a basis for determination of $\mathrm{dB} / \mathrm{dt}$ and will undergo close scrutiny.

o - Demonstrate that the maximum $\mathrm{dB} / \mathrm{dt}$ of the system is 6 T/s or less: Below level of concern."15

Options "B" and "C" are of no interest here since they permit rates of change above $6 \mathrm{~T} / \mathrm{s}$, while the maximum obtainable anywhere from a full size SMES is $3 \times 10^{-3} \mathrm{~T} / \mathrm{s}$.

Since exposure of personnel or animals to SMES fields could be for long periods of time, rather than for the periods of an MRI examination, which usually do not exceed one hour, we propose much more cautious procedures than advocated by the F.D.A. This approach is also advisable in view of the evidence published since 1985 on magnetochemical effects, ${ }^{16-19}$ which may - but not necessarily do - have some very subtle , and reversible (but not necessarily adverse) physiological effects in humans at flux density levels as low as $10^{-3} \mathrm{~T}(10 \mathrm{G})$.

Clearly constant fields below $10^{-3} \mathrm{~T}$ have no physiological effects on humans and large animals. Since field levels at the exclusion fence are only $10 \mathrm{G}$ and fall off to geomagnetic levels $(0.5 \mathrm{G})$ at a radius of $5400 \mathrm{~m}$, no precautions outside the exclusion fence are necessary other than possibly avoidance of 
superposition of above-ambient $60 \mathrm{~Hz}$ fields. The latter precaution is based on the suspicion that long duration exposure to simultaneously present $60 \mathrm{~Hz}$ and DC magnetic fields could have undesirable physiological effects.

Since the fields beyond the exclusion fence are also well below the 17 gauss level that may possibly affect cardiac pacers, even persons with such devices would not be at risk. Inside the exclusion fence it would be prudent to follow the Lawrence Livermore "guidelines", ${ }^{14}$ modified by lowering specified field levels for long duration exposure as indicted below and by adding additional guidelines to prevent long-term simultaneous exposure to DC and $60 \mathrm{~Hz}$ magnetic field exposure:

Maximum exposure - $2.0 \mathrm{~T}$ (maximum accessible level possible near the SMES coil)

Field less than $200 \mathrm{mT}$ - if the peak field to which workers are exposed is less than $200 \mathrm{mT}$, personnel may be exposed to a week long maximum average field strength of no more than 10 mT.

Fields greater than $200 \mathrm{mT}$ - if the peak exposure is greater than $200 \mathrm{mT}$, workers should be exposed to a daily maximum average field strength of no more than $10 \mathrm{mT}$ (same rationale as above).

\section{CONCLUSIONS REGARDING SMES OPERATIONS}

The proposed safety procedures which take into account not only what is known about biological effects of steady and slowly time varying DC magnetic fields, but also suspected (but presently not confirmed) effects of simultaneously present DC and $60 \mathrm{~Hz} \mathrm{AC}$ magnetic fields, would not affect normal operation of SMES units. Even the maximum contemplated rates of energy delivery produce rates of flux density change which give no health or safety concern and are entirely permissible under the proposed safety procedures.

Since the equipment in the inverter/recifier building would be operated automatically and since, in any case, exposure of personnel even inside that building - with the possible exception of very small areas - would be normally below $15 \mathrm{mT}$, the proposed safety procedures would not unduly restrict the operation of the ETM. In view of the concerns about simultaneous exposure to DC and $60 \mathrm{~Hz}$ magnetic fields it would be advisable for workers inside the rectifier/inverter building to wear one of the EPRI developed $60 \mathrm{~Hz}$ exposure meters $^{20}$ and to require that average daily exposure to $60 \mathrm{~Hz}$ magnetic fields be limited as much as possible.

In addition to health and personnel safety considerations, some other precautions are needed to prevent interference with aircraft navigational equipment and possible confusion to national magnetic measurement programs. In view of the magnetic field values, indicated in Fig.3, commercial, private and military aircraft should be excluded from a cylindrical region of $5400 \mathrm{~m}$ radius and $7000 \mathrm{~m}$ height above ground where the magnetic field would be above geomagnetic level. The Federal Aviation Agency, as well as appropriate offices in the U.S. Air Force, Navy, the U.S. Geological Survey and NASA should be made aware of the nature of the SMES facility and its exact location. Notification of the last two agencies is desirable, because they may at times carry out airborne geomagnetic surveys which would produce very strange data in the vicinity of SMES units.

\section{REFERENCES}

1. Stekly, Z. J. J., IDA/HQ 63-1412, Pulse Power Conference, February 1963, p. 53.

2. Sole, J., Comm. Energie Atomique, CEA-R3243, 1967.

3. Spiel, D. E. and Boom, R. W., Intemational Institute of Refrigeration, Low Temperatures and Electric Power, 158, 1969.

4. Irie, F. and Yamafuji, Y., ibid., 39.

5. Ferrier, M., ibid., 150.

6. Girard, B. and Sauzade, M., ibid, 158.

7. Thomas, D. B., Int. Col. Bubble Chambers, CERN 67-26, 1967.

8. Boom, R. W. and Peterson, H. A., IEEE Trans. Magnetics MAG-8, no. 3, 701-703, 1972.

9. Polk, C. and Boom, R.W., Adv. Cryo. Engr., Vol 31 (1986).

10. Peterson, H. A., Boom, R. W., USA patent $\# 4,122,512$, Oct 24 , 1978.

11. Moses, R. W., Adv. Cryo. Engn., Vol. 21 (1976).

12. Eyssa, Y. M., Mechanics of Superconducting Structures, ASME Publication AMD, Vol. 41, 67-68 (1980).

13. Eyssa, Y. M., J. Phys. D: Appl. Phys., Vol. 13, 1791 (1980).

14. Miller, G. (revised by), Health and Safety Manual, Supplement 26.12: Working in Magnetic Fields, Univ. of California-Lawrence Livermore National Laboratory (June 27, 1985).

15. U.S. FDA, 510 (K) Guidance: Safety Parameter Action Levels for MR Devices (August 1988).

16. Savitz, D. A., Wachtel, H. W, Bames, F. A., John, E. M. and Tvrdik, J. G., Amer. Journal Epidemiology, Vol. 128-1, 21-38 (1988).

17. Smith, S. D., McLeod, B. R., Liboff, A. R. and Cooksey, K., Pioelectromagnetics, Vol. 8, 215-227 (1987).

18. Pavlicek W., et al., Radiology, Vol. 147, 149 (1983).

19. Gaffey, C. T., Tenforde, T. S. and Dean, E. E., Bioelectromagnetics, Vol. 1, 209 (1980).

20. Sussman, S. S., EPRI Joumal, Vol. 13 (2), 50-52 (1988). 